\title{
The water-scarcity footprint of Australian adult diets - evidence from a large population health survey
}

\section{Abstract}

The food system is responsible for around $70 \%$ of global freshwater use. Pathways toward responsible consumption and production of food are therefore critically needed to ensure the planetary boundary for freshwater use is not transgressed. There is also an uneven spatial distribution of freshwater resources and human water demands, meaning that water-scarcity is acute in some regions but a lesser concern in others. Quantifying the water-scarcity impacts associated with food consumption is therefore a complex challenge due to the diversity of individual eating patterns, the very large number of individual food products available, and the many different regions where food is grown or processed. To our knowledge, this is the first study to calculate water footprints for a large number of self-selected diets. Life cycle assessment was used to model the water-scarcity footprints of 9,341 individual Australian adult diets obtained through 24-hour recall as part of the most recent Australian Health Survey. Three water-scarcity indicators were used, including the AWARE model recently developed by a project group working under the auspices of the United Nations Environment Programme (UNEP) / Society of Environmental Toxicology and Chemistry (SETAC) Life Cycle Initiative (www.lifecycleinitiative.org). In addition, a diet quality score was calculated for each of these diets. Our objective was to identify pathways toward healthier diets with lower water-scarcity impacts. Dietary water-scarcity footprints averaged $362 \mathrm{~L}^{-\mathrm{eq}}$ person $^{-1}$ day $^{-1}$ and were highly variable (sd. $218 \mathrm{~L}_{\text {-eq person }}^{-1}$ day $^{-1}$ ), reflecting the diversity of eating habits in the general community. The largest waterscarcity impacts were related to the excessive consumption of discretionary foods (alcoholic beverages, processed meat products, dairy desserts, cream, butter, muesli bars, confectionery, chocolate, biscuits, cakes, waffles, fried potato and extruded snacks, etc.). The potential to reduce dietary water-scarcity impacts is large, although the opportunity to intervene through amended dietary guidelines is not straightforward due to the large variations in water-scarcity footprint intensity between individual foods within a food group, and the inability of consumers to identify lower water-scarcity footprint products without food labeling. Reductions in the waterscarcity footprint of Australian food consumption are likely best achieved through reductions in food waste, technological change to improve water-use efficiency in food production, as well as the implementation of product reformulation and procurement strategies in the food manufacturing sector to avoid higher water-scarcity footprint intensity ingredients.

\section{Conflict of Interest}

This study was funded, in part, by a grant from Meat \& Livestock Australia (Sydney, Australia). The authors have exercised freedom in interpreting the data and making the decision to publish. 